ORIGINAL STUDY

\title{
Evolution of microbial etiology in acute and chronic rhinosinusitis and its role in the current management of antibiotic treatment
}

\author{
Iulia Sabaru ${ }^{1,2}$, Codrut Sarafoleanu ${ }^{1,3}$, Alina Maria Borcan ${ }^{1,4}$ \\ 1"Carol Davila" University of Medicine and Pharmacy, Bucharest, Romania \\ 2"C.I. Parhon" National Institute of Endocrinology, Bucharest, Romania \\ ${ }^{3}$ ENT\&HNS Department, "Sfanta Maria" Hospital, Bucharest, Romania \\ "’Prof. Dr. Matei Bals” National Institute for Infectious Disease, Bucharest, Romania
}

\section{ABSTRACT}

BACKGROUND. Acute and chronic rhinosinusitis (CRS) are common conditions worldwide. In most cases, the etiology of acute rhinosinusitis (ARS) is viral, but there can be cases complicated by bacterial infection. The bacterial pathogens responsible for acute bacterial rhinosinusitis (ABRS) in most cases are Streptococcus pneumoniae, Haemophilus influenza and Moraxella catarrhalis. In recent years, some changes regarding this issue have been communicated. Also, the pathophysiology of CRS becomes a problem due to the increasing percentage of resistant or recurrent cases.

OBJECTIVE. To identify the bacterial spectrum in patients diagnosed with ABRS and CRS and to establish the actual resistance rates of the most prescribed antibiotics for these affections in order to initiate the correct antibiotic treatment.

MATERIAL AND METHODS. We performed a prospective study on 40 adult patients with ABRS and 70 patients with CRS. The standard microbiological procedures were performed in order to identify the involved microorganisms. The Antibiotic Susceptibility Test of the clinical isolates was performed to routinely used antibiotics according to EUCAST.

RESULT. ABRS: A total of 21 types of pathogenic bacteria were isolated. The results indicated changes in the percentages of the traditionally involved bacteria, other species of streptococci and Staphylococcus aureus representing important pathogens. Almost half of the samples were polymicrobial. CRS: 12 bacteria were incriminated for CRS, Staphylococcus aureus and Pseudomonas aeruginosa being the most frequently identified pathogens. Regarding the antibiotic treatment, we established that in our country the resistance rates are higher than the ones communicated by WHO (especially for macrolides) and the fluoroquinolones seem to be the class with the highest safety profile.

CONCLUSION. Study results demonstrate some changes of the bacteriologic spectrum in ABRS in this geographic area. The pathogens responsible for CRS are found in approximately the same percentage as presented in other studies. Antibiotic treatment demands attention considering the increasing trend of antibiotic resistance of the bacteria causing ABRS and CRS.

KEYWORDS: ABRS, CRS, microbiologic diagnosis, antibiotic resistance.

\section{INTRODUCTION}

Rhinosinusitis can be defined as any inflammation of the nose and the paranasal sinus mucosa. The classification of rhinosinusitis includes: a) acute rhinosinusitis (ARS) - a new infection that may last up to four weeks; it can be viral (duration of symptoms for less than 10 days) or postviral/ bacterial (ABRS) - increase of symptoms after 5 days or persistent symptoms after 10 days, with less than 12-week duration; b) chronic rhinosinusitis (CRS) - involve more than 12 weeks of symptoms.

According to EPOS 2012, acute bacterial rhinosi- nusitis is defined "by the presence of at least 3 symptoms/signs of discoloured discharge and purulent secretion in cavum nasi, severe local pain (with unilateral predominance), fever $\left(>38^{\circ} \mathrm{C}\right)$, elevated $\mathrm{ESR} / \mathrm{CRP}^{1}$ " or a deterioration after an initial milder phase of illness. Regarding chronic rhinosinusitis (with or without nasal polyps), the clinical diagnosis involves "the presence of two or more symptoms one of which should be either nasal blockage/obstruction/congestion or nasal discharge (anterior/ posterior nasal drip)" associated or not with facial pressure, reduction or loss of smell - symptoms present for more than 12 weeks ${ }^{1}$. 
Paraclinically, endoscopic findings may be represented by: edema of the middle meatus mucosa, mucopurulent discharge primarily from the middle meatus or nasal polyps. Imaging shows mucosal changes within the ostiomeatal complex, edema of the mucosa and fluid accumulations in the sinuses and, sometimes, the effect of the above on the bone walls ${ }^{1}$.

The microbiology of acute and chronic rhinosinusitis is well known. In acute bacterial rhinosinusitis, the major pathogens are aerobic (Streptococcus pneumoniae, Haemophilus influenzae and Moraxella catarrhalis) and facultative anaerobic bacteria ${ }^{2}$. In chronic rhinosinusitis, Staphyloccocus aureus and anaerobic bacteria are the most frequently involved pathogens ${ }^{2,3}$.

Nowadays, more authors observe in their studies changes in the microbiological spectrum. Treatment failures, resistant cases or quick recurrences after treatment could be only a few of the responsible factors for the dynamics of rhinosinusitis microbiology, considering the problem of antibiotic resistance ${ }^{4}$. Also, the heptavalent conjugate pneumococcal vaccine has brought a number of changes.

Lately, there have been reported very high incidences as far as acute rhinosinusitis is concerned. It is estimated that adults suffer two to five episodes of viral ARS per year and only 0.5$2 \%$ of ARS are complicated by bacteria infection $^{1,5,6}$. Regarding the epidemiology of chronic rhinosinusitis, recent studies show that almost $15 \%$ of the population is affected. Those elevated levels of incidence are transforming rhinosinusitis in one of the most common diseases, and, at the same time, a very consuming financial resources disorder.

Thus, taking into account the incidence of this disease nowadays, the recent changes in the infectious etiology and the antibiotic resistance we are facing worldwide, we considered necessary a study that could reveal the actual bacterial spectrum in acute and chronic rhinosinusitis and also the correct antibiotic treatment.

\section{MATERIAL AND METHODS}

A clinical prospective study was performed between January 2014 and December 2017. The population of the study consisted of 40 adult patients diagnosed with ABRS and 70 patients with CRS. All the patients were clinically diagnosed and had endoscopic, imagistic and microbiologically confirmation of the disease.

The exclusion criteria were: nasal anatomic abnormalities; patients with complicated RS, with history of sinus surgery; patients with antibiotic treatment within the previous week; imunocompromised patients; patients with cystic fibrosis, with concurrent infections and/or neoplasm or positive history for drugs and alcohol abuse; patients with nosocomial infections. Also, those patients who had the inability to comply with the protocol of the study were excluded.

The clinical diagnosis was established according to the clinical criteria from EPOS 2012

The study protocol included 3 visits. The first visit consisted of the patients' informed consent for clinical study participation, Visual Analogue Scale (VAS) filling, clinical exam, endoscopic assessment and sampling from the middle or superior meatus, under direct endoscopic control. Samples were transported to the microbiology laboratory in less than one hour.

Bacterial Identification Samples were cultured in Blood agar, Eosin methylene blue agar and Chocolate agar, and incubated at $37^{\circ} \mathrm{C}$ for $18-24$ hours. The standard microbiological procedures were performed in order to identify the involved microorganisms.

The Antibiotic Susceptibility Test of the clinical isolates included the routinely used antibiotics according to the European Committee on Antimicrobial Susceptibility Testing (EUCAST).

Patients with ARS received medical treatment which consisted of NSAID (ibuprofen $400 \mathrm{mg}$ x2/ day), a herbal compound, an intranasal corticosteroid (mometasone furoate $50 \mu \mathrm{g} /$ nostril, twice a day), a nasal decongestant (recommended dose, if needed), nasal irrigations with hypertonic saline solution, mucolytics - for 10 days.

Patients diagnosed with CRS received, for 10 days initially, an intranasal corticosteroid (mometasone furoate $50 \mu \mathrm{g} /$ nostril, twice a day), nasal irrigation with isotonic or hypertonic saline solutions and a herbal compound or mucolytics, if rhinorrhea was the main symptom.

At the second visit (72 hours after the first visit), the patient received the antibiotic treatment according to the antibiogram results.

During the third visit (after 10 days of antibiotic treatment), the patients were reassessed by clinical examination. They underwent a nasal endoscopy assessment and a second sample was taken in order to confirm the microbiological healing. Also, the patients were kindly asked to fill again VAS (validating the clinical healing in ABRS and assessing the disease evolution in CRS).

Statistical data were analysed using the SPSS17 software. 


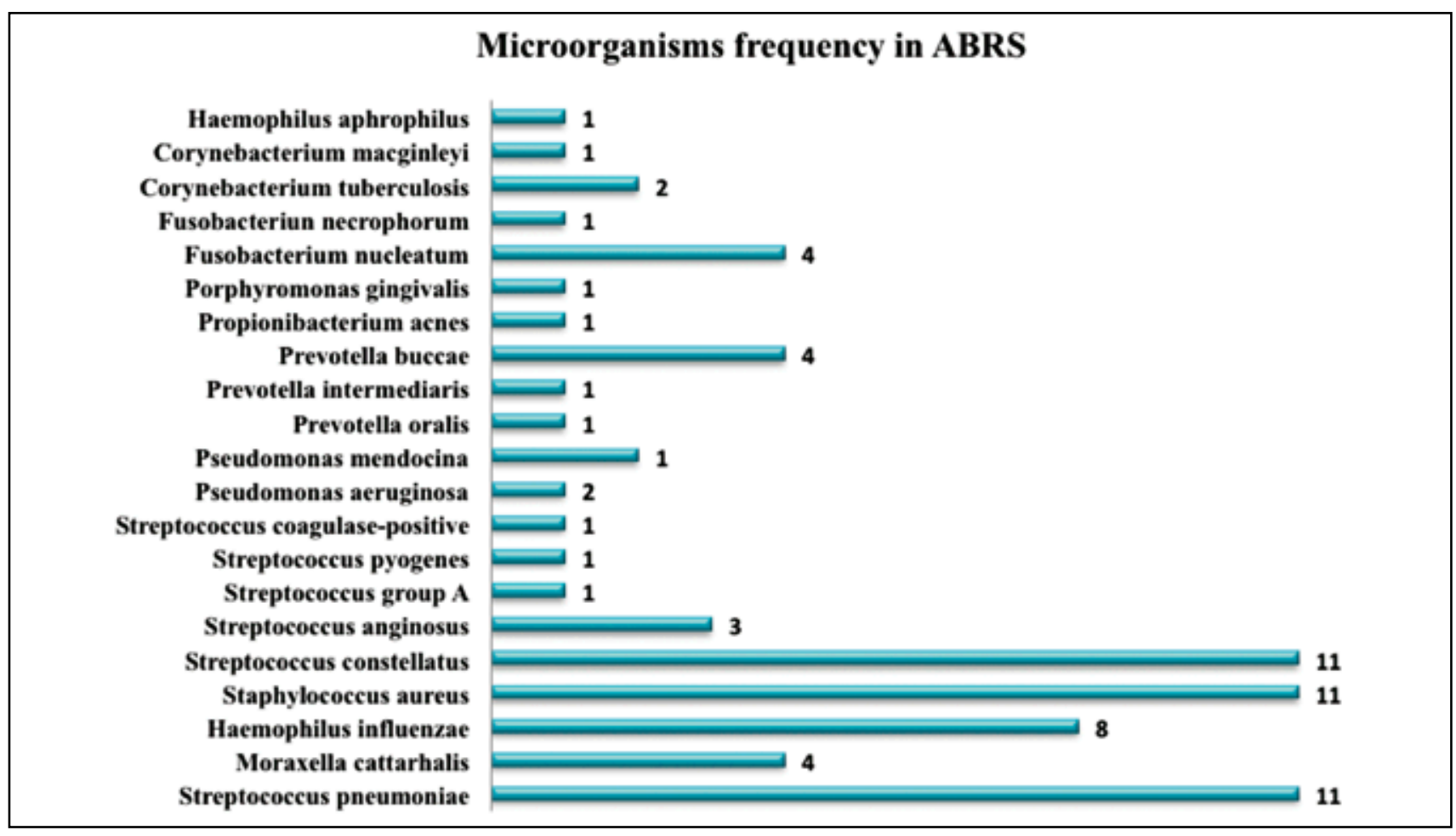

Figure 1 Microorganisms frequency in ABRS.

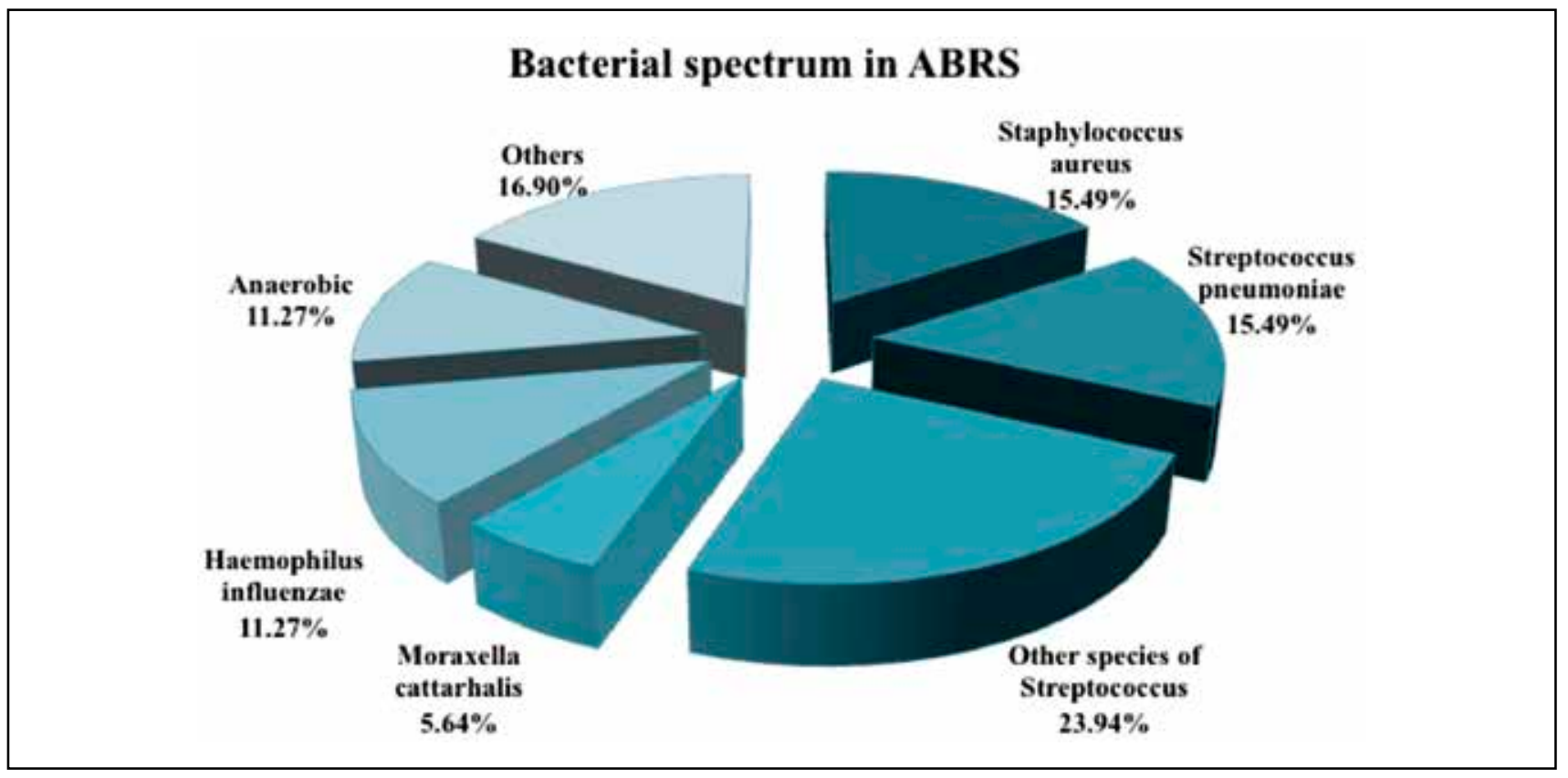

Figure 2 Bacterial spectrum in ABRS.

\section{RESULTS}

A total of 110 adult patients were included in our prospective clinical study. 40 of them were diagnosed with ABRS, while 70 were patients suf- fering from CRS. In the ABRS group, we had 27 males and 13 females. Concerning the patients diagnosed with CRS, 36 males and 34 females participated. Patients ages varied from 19 to 79 years old. 


\title{
Microorganisms frequency in CRS
}

\author{
Streptococcus pyogenes \\ Proteus mirabilis \\ Haemophilus parainfluenzae \\ Staphylococcus xylosus \\ Enterobacterium cloacae \\ Pseudomonas aeruginosa \\ Streptococcus constellatus \\ Haemophilus influenzae \\ Moraxella cattarhalis \\ Klebsiella pneumoniae \\ Streptococcus pneumoniae \\ Staphylococcus aureus
}

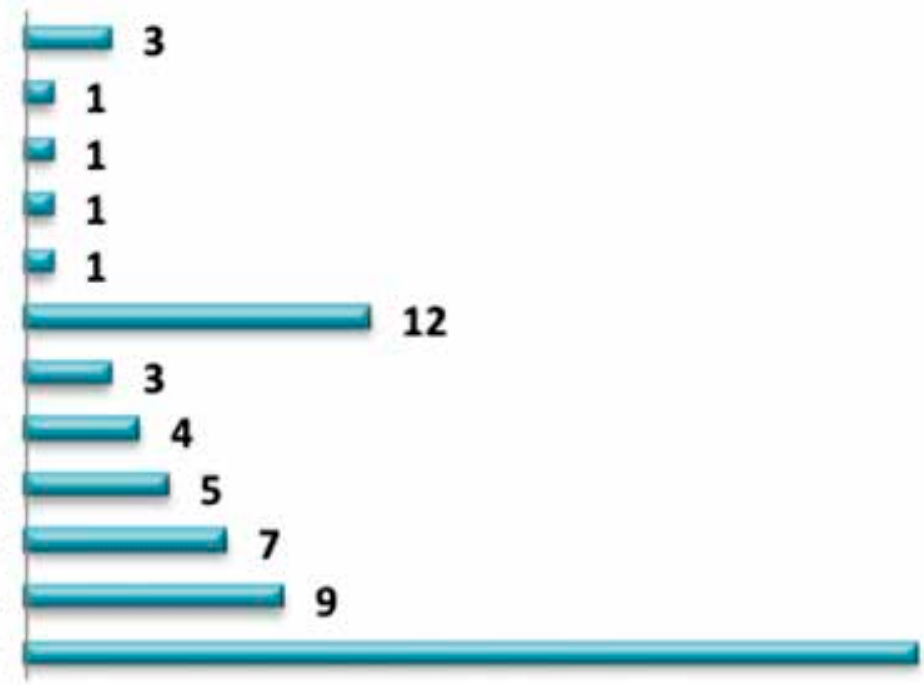

Figure 3 Microorganisms frequency in CRS.

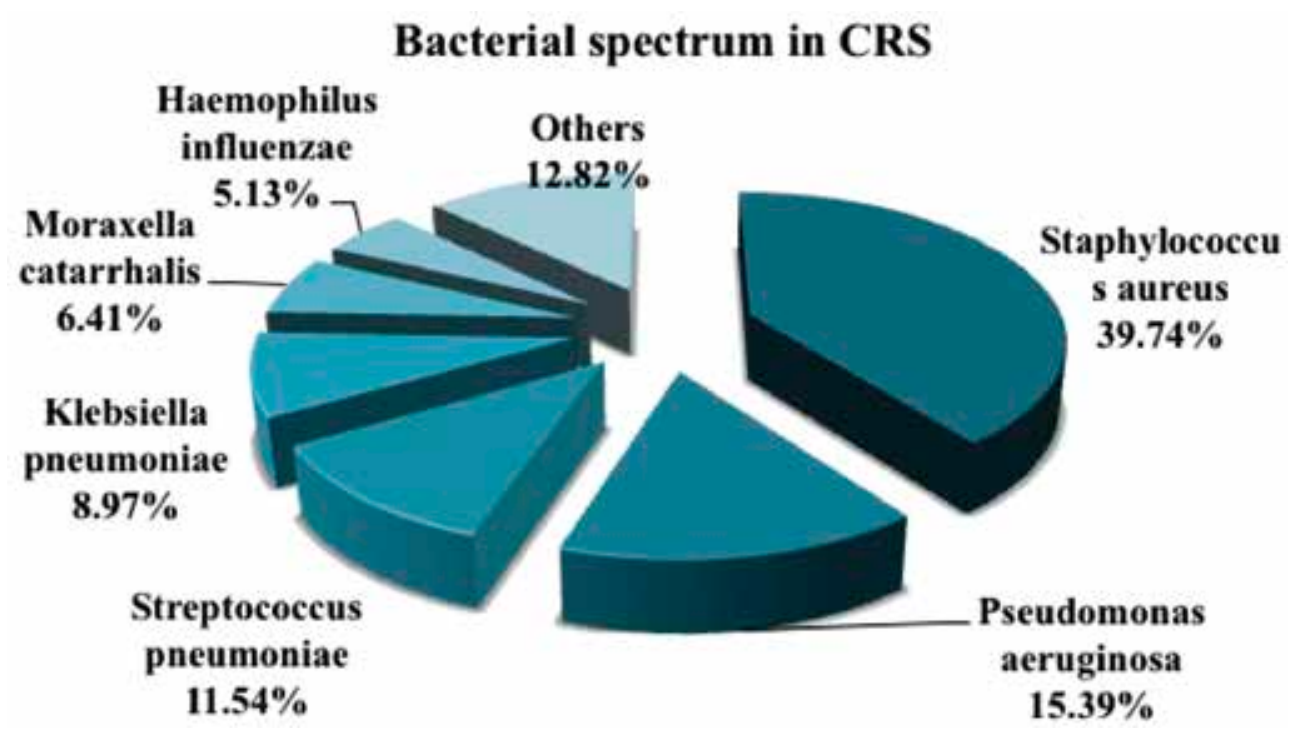

Figure 4 Bacterial spectrum in CRS.

\section{$\underline{\text { ABRS }}$}

In the ABRS group, 21 types of bacteria were identified (Figure 1). The most frequently encountered bacteria were: Streptococcus species $39.43 \%$ (Streptococcus pneumoniae representing $15.49 \%)$, Staphylococcus aureus $15.49 \%$, Morax- ella catarrhalis - $5.64 \%$, Haemophilus influenzae - $11.27 \%$ of cases (Figure 2). $11.27 \%$ of pathogens were anaerobic bacteria.

$40 \%$ of cultures were polymicrobial. During the microbiological examination, specimens with 2,3,4 and even 5 bacterial isolates were identified. 


\section{GRS}

When analysing the samples prelevated from patients diagnosed with CRS, we found 12 types of bacteria (Figure 3). The most often encountered bacteria were Staphylococcus aureus
$(39.74 \%)$, Pseudomonas aeruginosa $(15.38 \%)$, Streptococcus pneumoniae $(11.53 \%)$ and Klebsiella pneumoniae $(8.97 \%)$ (Figure 4 ).

8 samples were polymicrobial, but contained only 2 species. In 5 cases, one of them was Staph-

\section{Resistance of antibiotic therapy in ABRS}

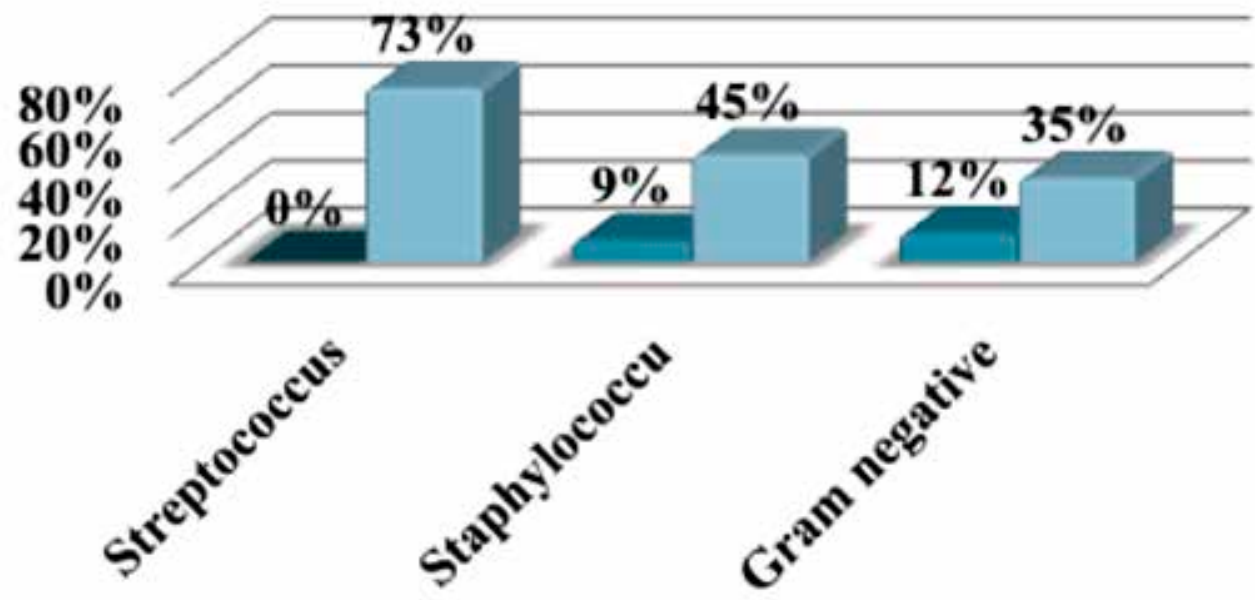

Flouroquinolones $\square$ Macrolides

Figure 5 Resistance of antibiotic therapy in ABRS.

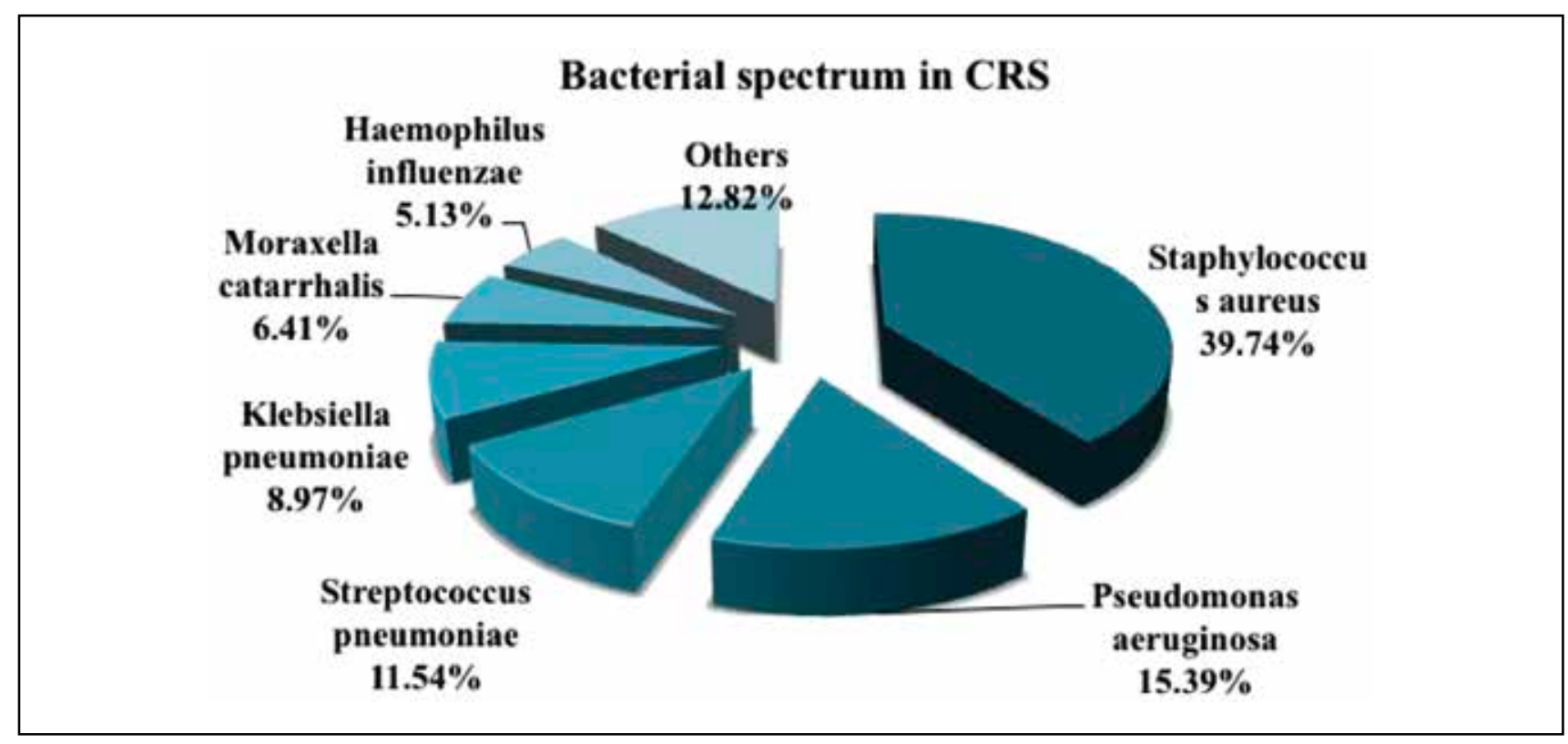

Figure 6 Resistance of antibiotic therapy in CRS. 
ylococcus aureus (associated in 3 cases with Pseudomonas aeruginosa and in 2 cases with Klebsiella pneumoniae), in 1 case Streptococcus constellatus with Staphylococcus xylosus coexisted, in 1 case Klebsiella pneumoniae with Proteus mirabilis and in another sample the two involved bacteria were Pseudomonas aureus and Enterobacter cloacae.

The bacterial susceptibility to different antibiotics was realized according to EUCAST recommendations, but in this article the data regarding the macrolides and fluoroquinolones rates of resistance are highlighted, considering that in Romania the two classes are the most frequently used in the rhinosinusitis treatment.

The conclusion was that the actual germs involved in ABRS and CRS have a significantly higher degree of sensitivity to the newer fluoroquinolones compared to macrolides (Figures $5,6)$.

Regarding the resistance rate of Streptococcus pneumoniae and Staphylococcus aureus to fluoroquinolones and macrolides, the analysis showed no significant differences when treating acute or chronic rhinosinusitis. In the Pseudomonas aeruginosa case, when involved in chronic disease, in $83 \%$ of the cases it is resistant to macrolides treatment, but using a fluoroquinolone represents a good decision in more than $90 \%$ of cases. Also, another important fact is that Haemophilus influenzae is susceptible to third-generation cephalosporin, and medical treatment using fluoroquinolones or macrolides is doomed to failure.

\section{DISCUSSIONS}

According to EPOS 2012, the most common bacteria causing ABRS include Streptococcus pneumoniae, Haemophilus influenzae, Streptococcus pyogenes, Moraxella catarrhalis and Staphylococcus aureus ${ }^{1,7}$.

However, in our study, we observe that the "infernal trio" was identified in only $32.39 \%$ of cases, the most frequently involved in ABRS 'etiology being other Streptococcal species $(23.94 \%)$. Brook and Gober reported a higher incidence of Streptococcus pneumonia (27\%) in descending trend, Haemophilus influenza (44\%) and Moraxella catarrhalis (14\%) with small increases ${ }^{8}$. They observe also a higher percentage of Streptococcal species responsible for acute rhinosinusitis, but smaller in comparison with our study results ${ }^{8}$. The authors considered that the introduction of the Pneumococcal con- jugate vaccine could be one of the causes that led to some changes in the bacterial spectrum of ABRS. Another vaccine was mentioned when pathogen profile of ABRS was discussed - H. influenzae type $\mathrm{B}$ (Hib) vaccine ${ }^{9}$. Mustafa and $\mathrm{Pa}$ tawari considered that $\mathrm{H}$. influenzae type $\mathrm{B}$ infections have dramatically decreased and nowadays the non-type $\mathrm{H}$. influenza (NTHI) is predominantly seen in the clinics ${ }^{9}$. However, in a study performed in Thailand in 2015, a high percentage of Haemophilus influenzae (25\%) was encountered when studying ABRS microbiome, but over the years the percentages varied from $20 \%$ to $72 \%{ }^{10}$.

Staphylococcus aureus represented an important pathogen in our clinical study, being responsible for ABRS in $16 \%$ of cases. Also, it was found coexisting in 7 of 17 polymicrobial cultures, highlighting, probable, a series of patientdependent immunological implications.

In our ABRS group, $40 \%$ polymicrobial cultures were found, a much more increased level compared with other studies $(17.3 \%)^{10}$. However, we excluded swab contamination with commensal flora (Staphylococcus aureus), considering that we reported, in another study, a correlation of $91.15 \%$ between sampling directly from the meatus under endoscopic control and sampling by sinusoscopy ${ }^{4}$.

Regarding the bacterial profile responsible for CRS, we found that Staphylococcus aureus $(39.74 \%)$, Pseudomonas aeruginosa (15.38\%), Streptococcus pneumoniae $(11.53 \%)$ and Klebsiella pneumoniae $(8.97 \%)$ are the most encountered pathogens. Most of these bacteria can be found in healthy patients as Kaspar et al. demonstrated in 2015, but only if the titer is low, are they considered to be resident flora ${ }^{11}$.

EPOS reveal also that $\mathrm{S}$. aureus is the most common traditional bacterial pathogen identified in CRS patients, but Gram-negative bacteria and anaerobes are also highly involved.

Unexpectedly, we have identified a smaller number of polymicrobial cultures in CRS patients' samples -8 , in which no more than 2 pathogens have been discovered. Also, Staphylococcus aureus was present in 5 from 8 samples (in 3 cases associated with Pseudomonas aeruginosa and in 2 cases with Klebsiella pneumoniae), the other associations being: Streptococcus constellatus with Staphylococcus xylosus, Klebsiella pneumoniae with Proteus mirabilis and in another sample - Pseudomonas aureus and Enterobacter cloacae.

Although in acute rhinosinusitis the percentage of polymicrobial cultures was almost $50 \%$, in 
chronic disease we must take into consideration the role of bacterial biofilms, which cannot be assessed by simple microbiological tests and require biopsy of the sinusal mucosa. It is well established that biofilvms are complex structures composed of bacteria and an extracellular protective layer. The bacterial biofilms are formed on the surface of the sinusal mucosa and represent a survival and, at the same time, an adaptive mechanism of bacteria. Biofilms serve to protect bacteria from both host defence mechanisms and antibiotics. Nowadays, biofilms are incriminated for the recurrent rhinosinusitis and for the resistant cases, even though the antibiotic treatment was prescribed in an empiric way or according to an antibiogram. Staphylococcus aureus, Streptococcus pneumonia, Pseudomonas aeruginosa, Haemophilus influenzae and Moraxella catarrhalis are only some of the multiple bacteria associated with CRS biofilms, so even if in the cultures fewer pathogens are found, the bacterial network is more complex and inaccessible when compared with the one involved in ABRS $^{12-16}$.

Concerning the antibiotic resistance, we all know the increased rates of amoxicillin - clavulanic acid (exceeding 50\%) communicated by the WHO and confirmed in our country in medical practice.

Thus, we tried to evaluate the resistance rates of macrolides and flouroquinolones when treating ABRS or CRS, considering the fact that in Romania, in most cases, the antibiotic treatment is empirically prescribed in the general practitioner's office.

In our study, the resistance rates of the most encountered bacteria involved in ABRS and CRS to macrolides exceed $25 \%$, the level communicated by WHO. The resistance percentages are similar for Staphylococcus aureus, Streptococcus pneumoniae and Pseudomonas aeruginosa, no matter if the affection is acute or chronic.

We observed that for Haemophilus influenzae, third-generation cephalosporins are the appropriate antibiotic treatment, fluoroquinolones or macrolides being ineffective.

We consider that fluoroquinolones were prescribed less than macrolides without a previous microbiological test in our country. Our results show resistance rates which do not exceed $12 \%$.

\section{CONCLUSIONS}

Acute and chronic rhinosinusitis are two of the most frequently diagnosed diseases, with impor- tant impact on patients` QoL and major economic implications.

One can observe a continuous tendency to change of the bacterial microorganisms involved in this pathology. These changes are, apparently, due to treatment failures, resistant cases and quick recurrence after treatment. Also, these problems related to antibiotic treatment appear because of the empiric way of prescribing antibiotic even if the rhinosinusitis has all the characteristics of a common cold, the administration of antibiotics without a previous antibiogram and the administration of antibiotic over a period of less than 10 days. The new vaccines addressed to Streptococcus pneumonia and Haemophilus influenza may also have an influence over the modified percentages in bacterial spectrum.

In ABRS, Streptococcus pneumoniae, Haemophilus influenzae, Moraxella catarrhalis are still the most common pathogens, but nowadays their frequencies are decreasing. Staphylococcus aureus is a major pathogen $(15.5 \%$ of cases of ABRS). Oral streptococci are implicated in almost $1 / 3$ of ABRS. In our findings, almost half of the cultures were polymicrobial; in $43.75 \%$ of these cases Staphylococcus aureus was identified.

In CRS, Staphylococcus aureus was the most encountered bacteria. Gram-negative bacteria were isolated in more than $50 \%$ of the samples, Pseudomonas aeruginosa being the most important.

Actual germs involved in ABRS and CRS have a significantly higher degree of sensitivity to the newer fluoroquinolones compared to other classes of antibiotics commonly used (e.g. penicillins, macrolides) in this pathology. So, this class could be still considered a safe alternative, but under the conditions of prudent and wellcalculated use. Otherwise, in the future, acute or chronic rhinosinusitis could become one serious health problem considering its incidence.

Conflict of interest: The authors have no conflict of interest.

Contribution of authors: All authors have equally contributed to this work.

\section{REFERENCES}

1. Fokkens WJ, Lund VJ, Mullol J, Bachert C, Alobid I, Baroody F, et al. EPOS 2012: European position paper on rhinosinusitis and nasal polyps 2012. Rhinology. 2012;50 (Suppl 23):1-298.

2. Jousimies-Somer HR, Savolainen S, Ylikoski JS. Bacteriological findings of acute maxillary sinusitis in young adults. J Clin Microbiol. 1988;26(10):1919-25.

3. Brook I. Discrepancies in the recovery of bacteria from multiple sinuses in acute and chronic sinusitis. Journal of Medical Microbiol- 
ogy. 2004;53:879-85. DOI: 10.1099/jmm.0.45655-0.

4. Sabaru I, Neagu A. Acute bacterial rhinosinusitis: new aspects regarding bacterial spectrum and microbiologic diagnosis. Romanian Journal of Rhinology. 2014;4(14):111-6.

5. Wang DY, Wardani RS, Singh K, Thanaviratananich S, Vicente G, Xu $\mathrm{G}$, et al. A survey on the management of acute rhinosinusitis among Asian physicians. Rhinology. 2011;49(3):264-71. DOI: 10.4193/ Rhino10.169.

6. Revai K, Dobbs LA, Nair S, Patel JA, Grady JJ, Chonmaitree T. Incidence of acute otitis media and sinusitis complicating upper respiratory tract infection: the effect of age. Pediatrics. 2007;119(6): e1408-12.

7. Udayasri B, Radhakumari T. Microbial etiology of chronic sinusitis. IOSR-JDMS. 2016;15(1):118-24

8. Brook I, Gober AE. Frequency of recovery of pathogens from the nasopharynx of children with acute maxillary sinusitis before and after the introduction of vaccination with the 7-valent pneumococcal vaccine. Int J Pediatr Otorhinolaryngol. 2007;71 (4):575-9. Epub 2007 Jun 30.

9. Mustafa M, Patawari P, Iftikhar HM, Shimmi SC, Hussain SS, Sien MM. Acute and chronic rhinosinusitis, pathophysiology and treatment. International Journal of Pharmaceutical Science Invention. 2015;4(2):30-6.

10. Thanaviratananich S, Chusakul S, Moungthong G, Luxameechan- porn T, Tantilipikorn P, Fooanant S, et al. A Surveillance Bacteriological Study of Acute Bacterial Rhinosinusitis in Thailand and the Clinical Responses to the Culture-directed Antibiotics. J Med Assoc Thai. 2015;98 Suppl 7:S204-16.

11. Pourmousa R, Dadashzadeh R, Ahangarkani F, Rezai MS. Frequency of Bacterial Agents Isolated From Patients With Chronic Sinusitis in Northern Iran. Glob J Health Sci. 2015;8(5):239-46. DOI: 10.5539/ gjhs.v8n5p239.

12. Suh JD, Cohen NA, Palmer JN. Biofilms in chronic rhinosinusitis. Curr Opin Otolaryngol Head Neck Surg. 2010;18(1):27-31. DOI: 10.1097/MOO.0b013e328334f670.

13. Suh JD, Ramakrishnan V, Palmer JN. Biofilms. Otolaryngol Clin North Am. 2010;43(3):521-30, viii. DOI: 10.1016/j.otc.2010.02.010.

14. Sanderson AR, Leid JG, Hunsaker D. Bacterial biofilms on the sinus mucosa of human subjects with chronic rhinosinusitis. Laryngoscope. 2006;116(7):1121-6.

15. Prince AA, Steiger JD, Khalid AN, Dogrhamji L, Reger C, Eau Claire $\mathrm{S}$, et al. Prevalence of biofilm-forming bacteria in chronic rhinosinusitis. Am J Rhinol. 2008;22(3):239-45. DOI: 10.2500/ ajr.2008.22.3180.

16. Foreman A, Psaltis AJ, Tan LW, Wormald PJ. Characterization of bacterial and fungal biofilms in chronic rhinosinusitis. Am J Rhinol Allergy. 2009;23(6):556-61. DOI: 10.2500/ajra.2009.23.3413. 